\title{
PI-RADS 2.1 - Image Interpretation: The Most Important Updates and Their Clinical Implications
}

\section{PI-RADS 2.1 - Befundinterpretation: Die wichtigsten Neuerungen und ihre klinischen Implikationen}

Authors

Thomas Beyer $^{1}{ }^{\circledR}$, Heinz-Peter Schlemmer ${ }^{2}$, Marc-André Weber ${ }^{1}{ }^{\mathbb{O}}$, Kolja M. $^{\text {Thierfelder }}{ }^{1}$

Affiliations

1 Institute of Diagnostic and Interventional Radiology, Pediatric Radiology and Neuroradiology, Rostock University Medical Center, Rostock, Germany

2 Radiology, German Cancer Research Centre, Heidelberg, Germany

Key words

prostate, PI-RADS, prostate cancer, uroradiology, MR imaging

received 04.03 .2020

accepted 10.11.2020

published online 21.12 .2020

Bibliography

Fortschr Röntgenstr 2021; 193: 787-795

DOI 10.1055/a-1324-4010

ISSN 1438-9029

(c) 2020. Thieme. All rights reserved.

Georg Thieme Verlag KG, Rüdigerstraße 14,

70469 Stuttgart, Germany

Correspondence

Dr. med. Thomas Beyer

Institute of Diagnostic and Interventional Radiology, Pediatric

Radiology and Neuroradiology, Rostock University Medical

Center, Ernst-Heydemann-Str. 6, 18057 Rostock, Germany

Tel.: +49/3 81/4949201

thomas.beyer@med.uni-rostock.de

\section{ABSTRACT}

Background Multiparametric magnetic resonance imaging (MRI) of the prostate plays a central role in the diagnosis of patients with suspected prostate cancer. The increasing distribution and application of the guideline for the standardization of image acquisition, evaluation, and reporting (Prostate Imaging - Reporting and Data System, PI-RADS), which was updated in 2019 to version 2.1, contributes to the success of the technique.

Materials and Methods The most important updates of PI-RADS version 2.1 presented in 2019 compared to the previous version PI-RADS 2.0 are highlighted and interpreted with regard to their clinical implications.
Results PI-RADS version 2.1 aims to simplify the application of the scoring scheme without changing the basic concept of dominant sequences (DWI in the peripheral zone, $\mathrm{T} 2$ in the transition zone). Of particular importance are the increasing role of diffusion-weighted imaging in the transition zone, the now mandatory high b-value of at least $1400 \mathrm{~s} / \mathrm{mm}^{2}$, and new information on the assessment of the central zone and the anterior fibromuscular stroma.

Conclusion PI-RADS version 2.1 published in 2019 addresses a number of changes to the previous version, including both the examination technique and image interpretation. Prospective clinical studies have yet to prove the extent to which the goals of reducing interreader variability and increasing the detection rate in the transition zone will be achieved.

\section{Key Points:}

- The new PI-RADS version 2.1. includes changes regarding image interpretation and examination technique

- The role of diffusion-weighted imaging is strengthened in the transition zone

- An ultra-high b-value of at least $1400 \mathrm{~s} / \mathrm{mm}^{2}$ is mandatory according to PI-RADS 2.1

- Biparametric MRI is not recommended for general application

\section{Citation Format}

- Beyer T, Schlemmer H, Weber M et al. PI-RADS 2.1 - Image Interpretation: The Most Important Updates and Their Clinical Implications. Fortschr Röntgenstr 2021; 193: 787795

\section{ZUSAMMENFASSUNG}

Hintergrund Die multiparametrische Magnetresonanztomografie (MRT) der Prostata übernimmt inzwischen eine zentrale Rolle in der Diagnostik bei Patienten mit Verdacht auf Prostatakarzinom. Zum Erfolg der Methode trägt die zunehmende Verbreitung der zuletzt im Jahr 2019 auf die Version 2.1 aktualisierte Leitlinie zur Standardisierung von Bildakquisition, Auswertung und Befundung (Prostate Imaging - Reporting and Data System, PI-RADS) bei.

Material und Methoden Die zentralen Neuerungen der im Frühjahr 2019 vorgestellten PI-RADS-Version 2.1 gegenüber der Vorgängerversion PI-RADS 2.0 werden vorgestellt und bezüglich ihrer klinischen Implikationen diskutiert. 
Ergebnisse Die PI-RADS-Version 2.1 zielt darauf ab, die Anwendung zu vereinfachen, ohne dabei das Grundkonzept der dominanten Sequenz (DWI in der peripheren Zone, T2 in der Transitionszone) zu ändern. Von besonderer Bedeutung sind die stärkere Gewichtung der Diffusionsbildgebung in der Transitionszone, der nun obligatorische hohe b-Wert von mindestens $1400 \mathrm{~s} / \mathrm{mm}^{2}$ und neue Hinweise zur Beurteilung der zentralen Zone und des anterioren fibromuskulären Stromas.
Schlussfolgerung Die 2019 veröffentlichte PI-RADS-Version 2.1 adressiert verschiedene Änderungen, die sowohl Untersuchungstechnik als auch Befundung betreffen. Inwieweit sich damit die Ziele der Reduktion der Interreader-Variabilität und der Erhöhung der Detektionsrate in der Transitionszone erreichen lassen, müssen prospektive klinische Studien zeigen.

\section{Introduction}

After lung cancer, prostate cancer is globally the second most common malignant non-cutaneous tumor disease in men. With $1,276,105$ new cases worldwide in 2018 , it was responsible for $3.8 \%$ of all cancer-related deaths in men [1]. In recent years, multiparametric magnetic resonance imaging (mpMRI) of the prostate has become significantly more important for the diagnosis of this disease and has become an important part of current European guidelines [2]. mpMRI of the prostate not only improves diagnosis and local staging but also facilitates prognosis prediction and treatment individualization [3, 4].

In 2012, the guidelines of the European Society of Urogenital Radiology (ESUR) provided for the first time consensus-based recommendations for standardizing the acquisition, evaluation, and reporting of prostate MRI [5]. These guidelines, which are named after the Prostate Imaging - Reporting and Data System (PI-RADS), were first revised in 2015 (PI-RADS 2.0) [6].

PI-RADS 2.0 simplified the concept of the dominant sequence for the first time (DWI in the peripheral zone, $T 2$ in the transition zone). On the whole, the new version was well received. However, studies continue to have a relatively low detection rate for carcinomas in the transition zone compared to the peripheral zone and a relatively high interreader variability, which can be attributed in part to ambiguous formulations in PI-RADS version 2.0 [711]. Under consideration of these issues and to incorporate technical advances, the AdMeTech Foundation, the ESUR, and the American College of Radiology (ACR) as the PI-RADS Steering Committee published PI-RADS version 2.1 in the spring of 2019 [12].

The updated version implements changes to minimize uncertainties in scoring and to reduce interreader variability without fundamentally changing the established application algorithm [13]. This article provides an overview of the most important changes in PI-RADS version 2.1 and discusses possible clinical implications.

\section{Changes regarding image acquisition}

As in version 2.0, revised PI-RADS version 2.1 also recommends the use of multiparametric MRI (mpMRI) from T2-weighted (T2w), diffusion-weighted imaging (DWI), and dynamic contrastenhanced (DCE) sequences. The advantages and disadvantages of biparametric MRI (bpMRI), which does not use contrast-enhanced sequences, are discussed in the guidelines, but the method is not recommended as the standard.

Revised version 2.1 also still recommends the use of MRI with a field strength of 1.5 and 3 Tesla. PI-RADS version 2.1 also does not provide a general recommendation regarding the use of endorectal coils, particularly since current scanners at both field strengths can ensure an adequate signal-to-noise ratio even without their use.

\section{T2-weighted imaging}

T2w remains the dominant sequence for evaluating the transition zone. While PI-RADS 2.0 still recommended the acquisition of T2-weighted sequences on all three standard planes (axial, sagittal, coronal), T2w on the axial plane as well as at least one additional orthogonal plane is sufficient according to PI-RADS 2.1. Even though PI-RADS 2.1 does not explicitly favor the sagittal or the coronal plane, the combination of at least transverse and sagittal T2w is indirectly advocated based on the recommended determination of the prostate volume using the sagittal plane.

Due to possible partial volume effects, the authors feel that acquisition of $\mathrm{T} 2 \mathrm{w}$ in all three standard planes is advantageous as long as time allows. This is particularly true with respect to the differentiation between typical and atypical BPH nodules based on the completeness of the T2 hypointense encapsulation of the nodule in the transition zone (see below) as newly added to PI-RADS 2.1. In addition to the two-dimensional T2-weighted sequences, 3D-T2w images can be useful for obtaining a better anatomical overview. However, the soft-tissue contrast is inferior to 2D-T2w images in some cases.

In contrast to PI-RADS 2.0, version 2.1 provides a recommendation regarding the orientation of the axial (T2w) sequence. The orientation should either be straight axial to the patient (regardless of the position of the prostate) or in an oblique axial plane perpendicular to the long axis of the prostate (perpendicular to the max. extension of the prostate between the base and the apex on the sagittal plane). The latter approach has the advantage that it typically facilitates fusion with ultrasound images during transrectal biopsy.

\section{Diffusion-weighted imaging}

Diffusion-weighted imaging (DWI) remains the dominant sequence for evaluating the peripheral zone.

Since ADC values can be subject to certain fluctuations as a function of the underlying b-values, PI-RADS 2.0 provided recom- 

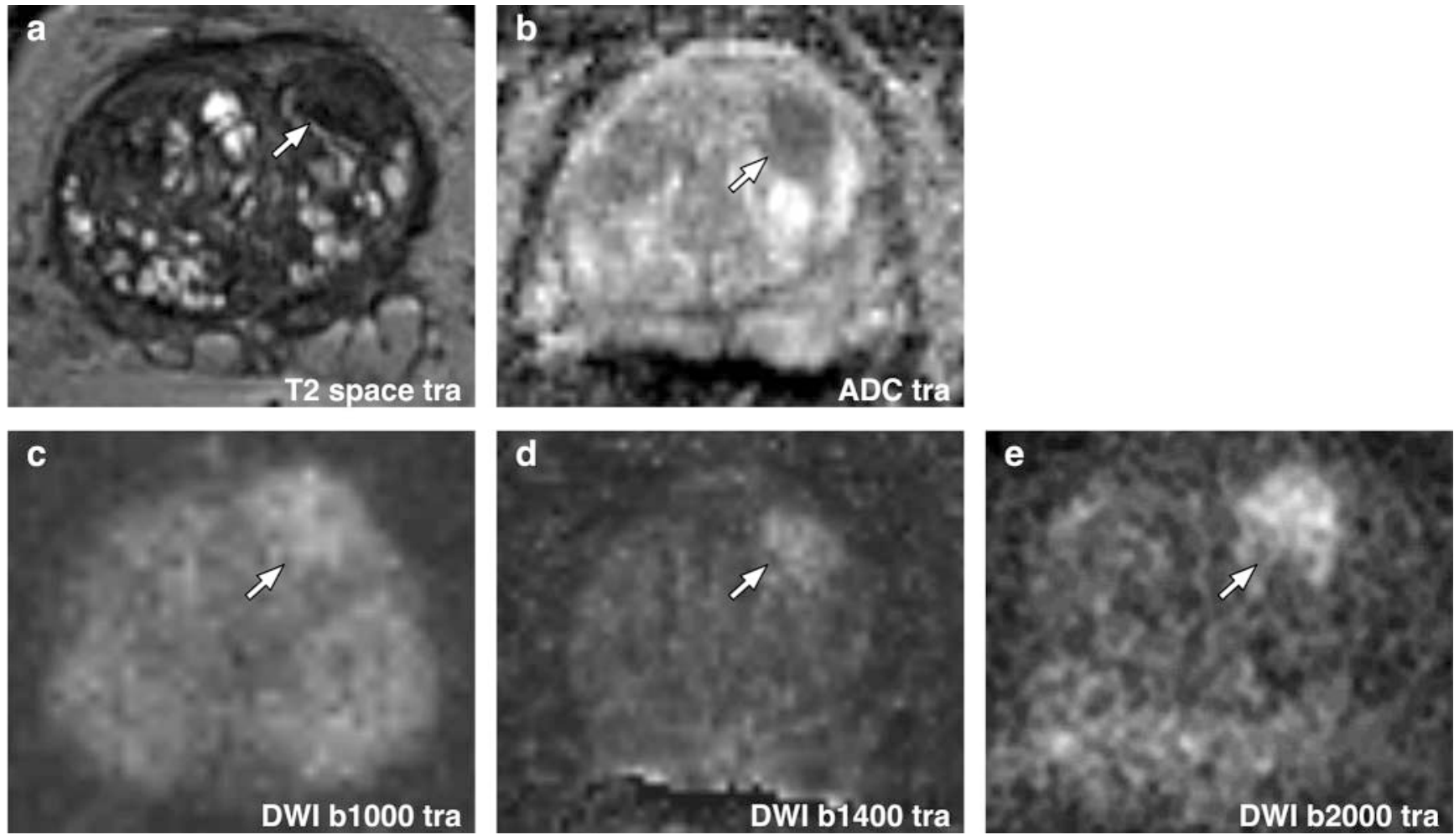

> Fig. 1 Different b-values in a biopsy-naïve 80 -year-old man with a PSA of $6.01 \mathrm{ng} / \mathrm{ml}$. Low T2w signal a at the base in the anterior transition zone on the left with restricted diffusion on ADC maps $\mathbf{b}$. The b1000 image $\mathbf{c}$ shows only a slight signal elevation in the corresponding area, which can be more clearly delimited from the surroundings in the b1400 image $\mathbf{d}$. The calculated b2000 image e shows the lesion most clearly. The $1.7 \mathrm{~cm}$ lesion corresponds to a PI-RADS 5 finding. The biopsy revealed a carcinoma with a Gleason score of $3+4=7 \mathrm{a}$.

mendations to keep these variations as minimal as possible. Therefore, PI-RADS 2.0 recommended the acquisition of low b-values between 50 and $100 \mathrm{~s} / \mathrm{mm}^{2}$ to avoid pseudoperfusion effects at a b-value of $0 \mathrm{~s} / \mathrm{mm}^{2}[14,15]$. Since the technical requirements of PI-RADS 2.0 are more difficult to meet in some cases, PI-RADS 2.1 allows a low b-value of $0 \mathrm{~s} / \mathrm{mm}^{2}$. To avoid kurtosis effects, $1000 \mathrm{~s} / \mathrm{mm}^{2}$ should be the maximum b-value used to calculate ADC $[16,17]$.

Apart from DWI with at least two b-values between 0 and $1000 \mathrm{~s} / \mathrm{mm}^{2}$ for the ADC calculation, which should always be performed, it has been able to be shown many times that "ultra-high" b-values can additionally reduce the $\mathrm{T} 2$ shine-through effect and increase the contrast between tumor and normal prostate tissue $[18,19]$ ( $\vee$ Fig. 1 ). Therefore, PI-RADS version 2.1 requires an ultra-high b-value of at least $1400 \mathrm{~s} / \mathrm{mm}^{2}$ (a) either extrapolated from the acquired lower b-value data (b0 to b1000) used to create the ADC map or (b) additionally measured separately. Since the detection of prostate cancer has improved significantly [20-23], the introduction of mandatory ultra-high b-values in PI-RADS 2.1 is definitely a useful update.

It is important when selecting the ultra-high b-value to take the field strength of the MRI system into consideration. As a rule, higher $\mathrm{b}$-values are possible at 3.0 $\mathrm{T}$ than $1.5 \mathrm{~T}$ under otherwise identical conditions [24]. Clinical practice in the coming years will show whether the introduction of the mandatory ultra-high b-value also proves to be technically feasible in the case of less powerful MRI systems.

\section{Dynamic contrast-enhanced sequence}

For image acquisition after i. v. contrast administration, PI-RADS 2.1 recommends the use of three-dimensional $\mathrm{T} 1$ sequences and highlights the advantages of a high spatial resolution compared to 2D-T1 sequences. DCE can help to better differentiate periprostatic veins from small carcinomas near the pseudocapsule [25].

Since temporal resolution during acquisition always entails compromises regarding spatial resolution, version 2.1 recommends a temporal resolution of up to 15 seconds (PI-RADS 2.0: $\leq 10 \mathrm{~s} ;<7 \mathrm{~s}$ preferred), since the possible risk of missing early contrast enhancement of lesions is negligible here [26]. To simplify the evaluation of dynamic contrast-enhanced sequences as much as possible, PI-RADS recommends a simple qualitative visual evaluation of DCE and does not require a quantitative evaluation. As in the previous version, PI-RADS 2.1 recommends a minimum examination time of 2 minutes after the administration of contrast agent.

\section{Multiparametric or biparametric MRI?}

In recent years there has been increasing interest in biparametric MRI (bpMRI) of the prostate, i. e., limitation to T2 and DWI sequences. Studies show that bpMRI has yielded good results in large centers [27, 28]. bpMRI is also faster and more cost-effective than mpMRI, a fact that must be taken into consideration in light of the significantly increasing number of cases [29]. Moreover, since contrast agents containing gadolinium are not used, the 
possible risks associated with these contrast agents are also eliminated.

In PI-RADS 2.1 the PI-RADS steering committee voices an opinion regarding the role of bPMRI for the first time but does not recommend it for general use. One reason for this is studies showing a higher sensitivity of mpMRI as a result of DCE-MRI [30-34]. This is particularly true in settings with less experienced evaluators. In addition, bPMRI relies completely on high-quality T2 and DWI thereby losing DCE-MRI as an important "back-up sequence" in cases of suboptimal DWI ( $\triangleright$ Fig. 2). The elimination of DCE-MRI can also result in more lesions being classified as PI-RADS 3 than PI-RADS 4 [35]. A high number of PI-RADS 3 lesions is problematic since this categorization does not include a standardized approach for a further course of action. In the absence of DCE-MRI, proponents of bpMRI are considering making the clinical management of category 3 lesions dependent on lesion volume. Patients with a PI-RADS 3 lesion with a volume of $<0.5 \mathrm{~cm}^{3}$ would undergo PSA follow-up and annual bpMRI examination and those with a lesion volume of $>0.5 \mathrm{~cm}^{3}$ would undergo targeted biopsy [36, 37].

The authors agree with the recommendation of the PI-RADS steering committee that mpMRI should be given preference when the priority of the examination is to make sure that no clinically significant prostate cancer is missed. bpMRI should initially be reserved for qualified centers and specialized radiologists with extensive experience and its role should be further evaluated in studies.

Summary of the most important changes regarding image acquisition according to PI-RADS 2.1

- T2w images should be acquired in axial orientation and at least one other orthogonal plane.

- Axial T2w images should be obtained either straight axial to the patient or in an oblique axial plane perpendicular to the long axis of the prostate.

- Acquisition of low b-values between 0-100 s/mm² $\left(50-100 \mathrm{~s} / \mathrm{mm}^{2}\right.$ is preferred) is now also possible.

- The maximum high b-value for ADC calculation is $\leq 1000 \mathrm{~s} / \mathrm{mm}^{2}$.

- A high b-value of at least $1400 \mathrm{~s} / \mathrm{mm}^{2}$ is either to be extrapolated or to be additionally measured separately.

- After i.v. administration of contrast agent, 3D-T1w sequences are to be given preference over 2D-T1w sequences.

- In the case of acquisition of dynamic contrast-enhanced sequences, a temporal resolution of up to 15 seconds is possible.

- bpMRI is currently not yet recommended for general use.

\section{Changes regarding interpretation of findings}

While the interpretation algorithm established in PI-RADS 2.0 relates exclusively to lesions in the peripheral zone and the transition zone, the anterior fibromuscular stroma (AFMS) and the central zone are additionally included as relevant regions in PI-RADS 2.1. However, these regions do not have their own scoring system and unremarkable findings do not require separate mention in the radiology report. In addition, there are small but clinically relevant
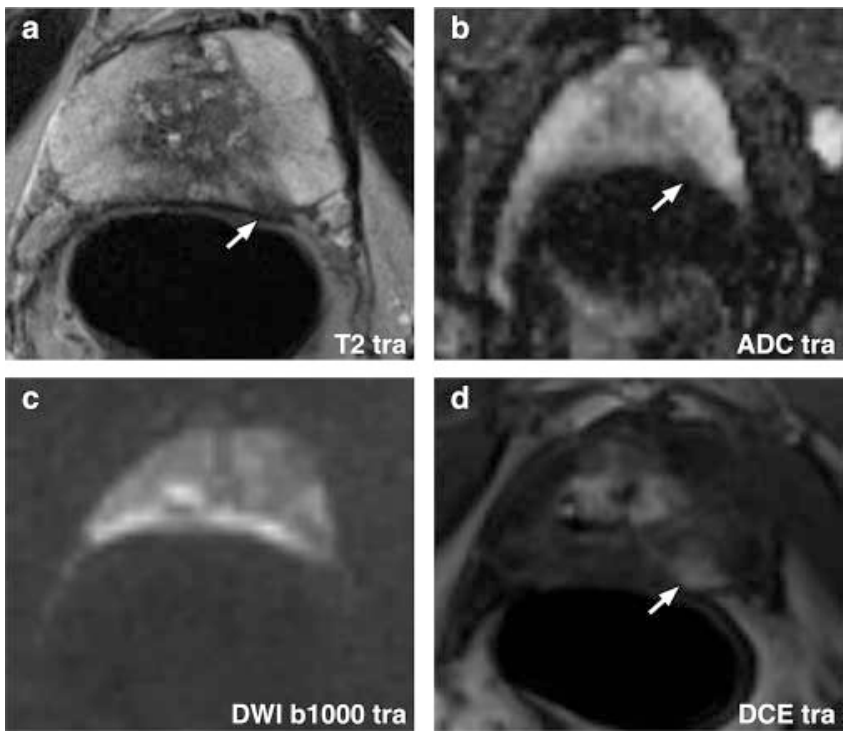

- Fig. 2 DCE-MRI as a "backup sequence" for DWI of low quality. A 77-year-old male with a PSA of $4.7 \mathrm{ng} / \mathrm{ml}$ after two negative biopsies. Focal blurred low T2w signal a. Artifacts due to abundant air in the rectum distort diffusion imaging. While focally reduced signal on ADC maps in this area $\mathbf{b}$, the $\mathbf{b} 1000$ image $\mathbf{c}$ cannot be evaluated diagnostically for this region. In this case, a contrastenhanced sequence $\mathbf{d}$ shows corresponding focal early arterial enhancement (arrow) - the lesion is upgraded to a PI-RADS category 4. The biopsy revealed prostate carcinoma with a Gleason score of $3+4=7$ a. Note: This example clearly shows that rectal voiding prior to examination contributes decisively to technical success and high diagnostic accuracy.

changes regarding the scoring of lesions in the transition zone and the interpretation of DWI and DCE-MRI.

\section{Central zone}

The central zone (CZ) is histologically very similar to the seminal vesicles and encircles the ejaculatory duct while extending from the base of the prostate dorsal to the TZ caudally in the direction of the prostate apex to the seminal colliculus (verumontanum). Due to its V-shaped configuration, the CZ can often be better identified on the coronal plane in up to $93 \%$ of mpMRI examinations [38, 39].

The normal $C Z$ is a region with bilaterally symmetric low signal intensity in T2w and ADC and is mildly hyperintense on DWI. Due to the histological similarity of the $C Z$ with the seminal vesicles and the low incidence of cancer of the seminal vesicles, cancer in the $C Z$ is also extremely rare and accounts for less than $5 \%$ of all cases of prostate cancer. Its etiological and locoregional connection with the seminal vesicles explains why the seminal vesicles are often infiltrated by tumors of the CZ [40]. Tumors of the CZ are often seen as asymmetrical masses with a hypointense signal on T2w, a hyperintense signal on DWI, and early enhancement on DCE-MRI. By discussing this anatomical region of the prostate and its physiological signal behavior, PI-RADS 2.1 aims to avoid possible misinterpretation of the normal CZ as cancer and to prevent misinterpretation of tumors near the base at the junction between the peripheral zone and the transition zone as the $C Z$. 


\section{PI-RADS 2.1 assessments}

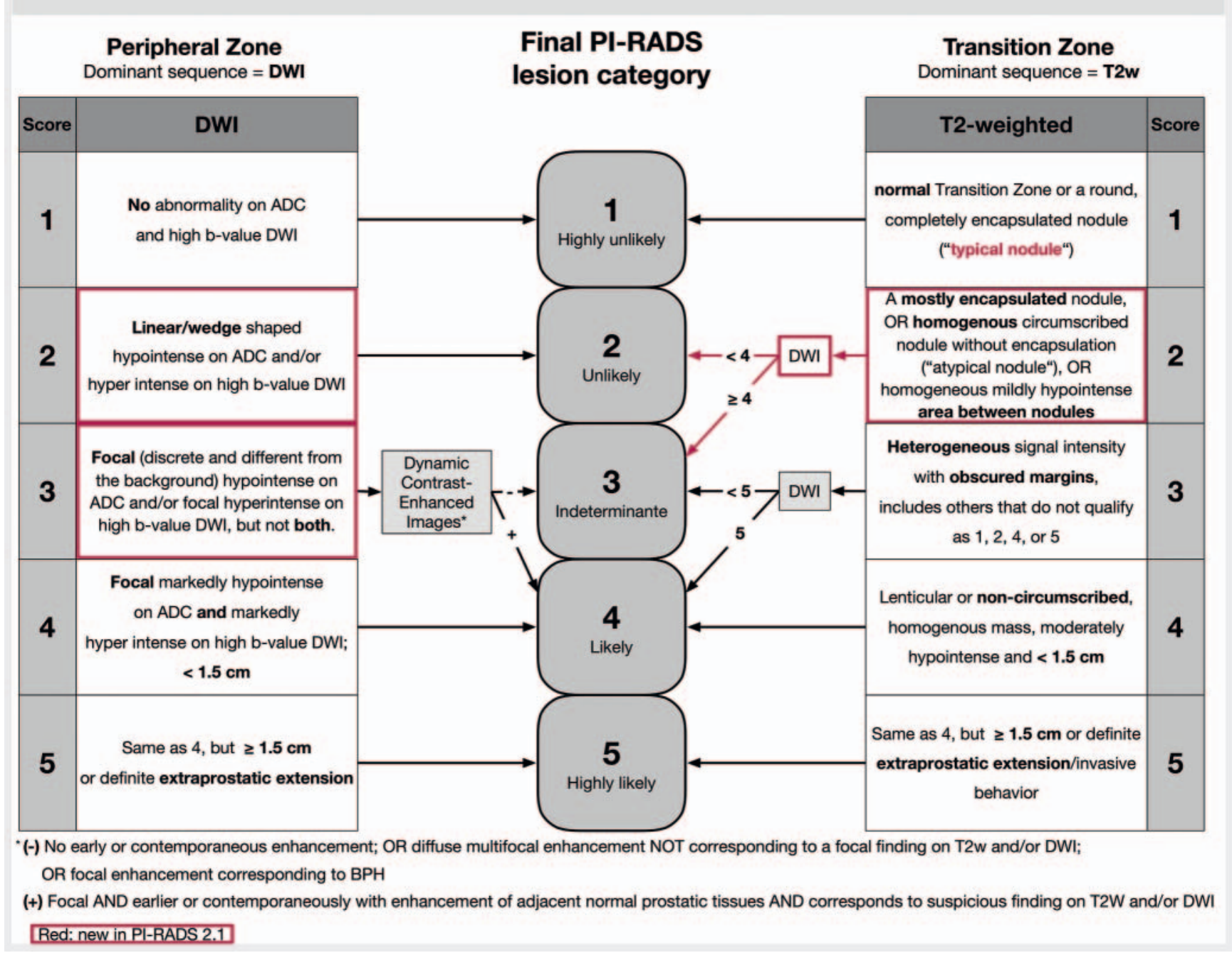

- Fig. 3 Reporting scheme according to the current PI-RADS version 2.1 The changes to PI-RADS version 2.0 are highlighted by a red frame.

\section{Anterior fibromuscular stroma}

Like the CZ, the normal anterior fibromuscular stroma (AFMS) is specifically discussed in PI-RADS 2.1 since it can be difficult to differentiate from ventral tumors [41]. It is comprised of vertically running muscle bundles and connective tissue and forms the ventral margin to the prostate as non-glandular tissue. The bilaterally symmetrical AFMS therefore has a low signal intensity on $\mathrm{T} 2 \mathrm{w}$, DWI, and ADC and delayed contrast enhancement ("low on all sequences") [13]. Cancers do not originate in the AFMS but can infiltrate the AFMS from the transition zone or the peripheral zone [42]. The evaluation criteria for lesions in the AFMS are therefore to be selected according to the region of origin (PZ or TZ). However, since this cannot always be definitively determined, there is a certain degree of diagnostic uncertainty in such cases which is recognized by the PI-RADS steering committee as a limitation of the assessment system.

\section{Transition zone}

Approximately $30 \%$ of all prostate cancers develop in the transition zone (TZ) [42]. It is known that the sensitivity and specificity of mpMRI for cancers in the TZ are lower than in the PZ [43]. BPH nodules of various morphology and levels of signal intensity, cystic changes, and stromal changes with an inhomogeneous T2 signal are regularly seen in the TZ [44]. Since "typical" BPH nodules (see below) are normally seen in the TZ in patients with suspicion of prostate cancer, PI-RADS version 2.1 reclassified "typical" BPH nodules in the TZ from a PI-RADS score of 2 (in version 2.0) to a PI-RADS score of 1 (in version 2.1) (see diagram in $>$ Fig. 3).

Typical BPH nodules are lesions that are fully encapsulated on T2w ( $\triangleright$ Fig. 4, 5a). Experience in the clinical routine with PI-RADS 2.1 and studies will show whether a more precise definition regarding the capsule is necessary, e. g. definition of the number of planes on which a complete capsule must be visible. 


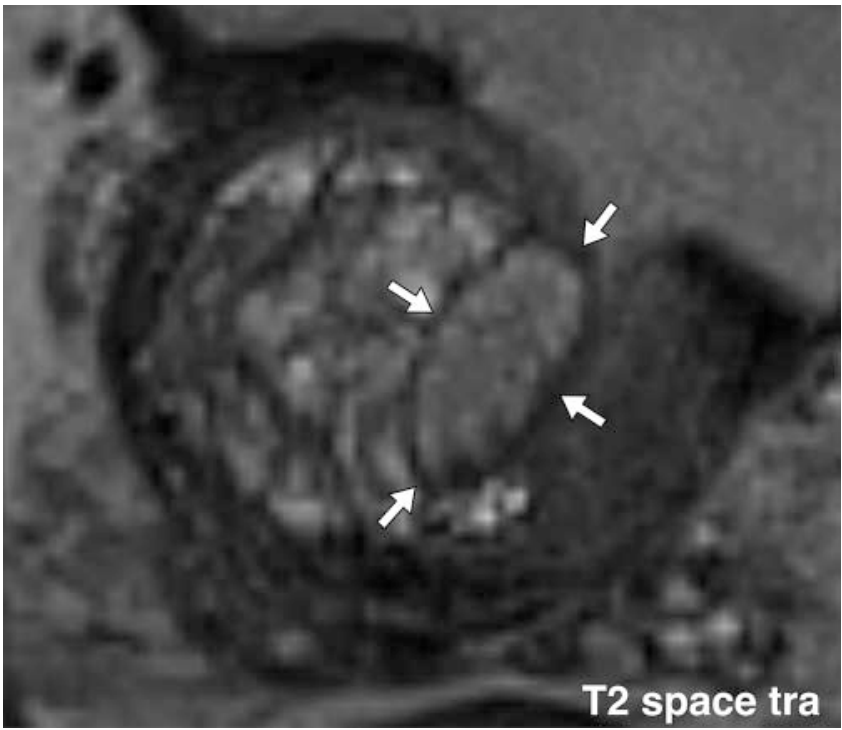

- Fig. 4 "Typical nodule" in transition zone (TZ) in a 60-year-old man with PSA increase to $6.38 \mathrm{ng} / \mathrm{ml}$ and negative biopsy. Oval, fully encapsulated (arrows) BPH-nodule in T2w within the left TZ. ADC maps and the high b-value DWI images (not shown) revealed no findings. According to PI-RADS 2.1, this is a PI-RADS 1 finding, which therefore does not usually need to be mentioned separately in the findings.

In contrast, atypical BPH nodules are assigned a PI-RADS score of 2 and are defined as nodules with the following characteristics:

- Mostly (but not completely) encapsulated ( $>$ Fig. 5b)

- Homogeneous, hypointense, circumscribed, without encapsulation ( $\triangleright$ Fig. $5 c$ )

- Homogeneous mildly hypointense area between nodules $(\triangleright$ Fig. 5d)

The presence of microcysts in the $\mathrm{BPH}$ nodule is also discussed in PI-RADS 2.1, is classified as a benign change [45], and is assigned a PI-RADS score of 1 (completely encapsulated nodule) or 2 (mostly but not completely encapsulated nodule).

PI-RADS 2.1 uses or more detailed and more differentiated description of changes in the T2 categories PI-RADS 1 and 2 to take into account the many different nodular changes in these categories (see $>$ Fig. 3 ).

In addition to the revised T2 scoring in the TZ, PI-RADS 2.1 assigned greater value to diffusion imaging in the TZ: Atypical nodules in the TZ (PI-RADS score 2 see above) can now to be upgraded via DWI to a total PI-RADS score of 3 in the case of diffusion restriction with a score $\geq 4$ (see $>$ Fig. $\mathbf{3}, \mathbf{6}$ ). An upgrade from a T2w score of 3 to a total PI-RADS score of 4 can still only be achieved in the case of a DWI score of 5 (see $>$ Fig. 3 ).

The greater inclusion of DWI in PI-RADS 2.1 is intended to increase the sensitivity of the detection of lesions in the TZ. However, this will only be the case if corresponding PI-RADS 3 lesions are also biopsied in a targeted manner. Initial results indicate that prostate cancers in the TZ can be detected significantly better using PI-RADS 2.1 than PI-RADS 2.0 with simultaneously reduced interreader variability [44].
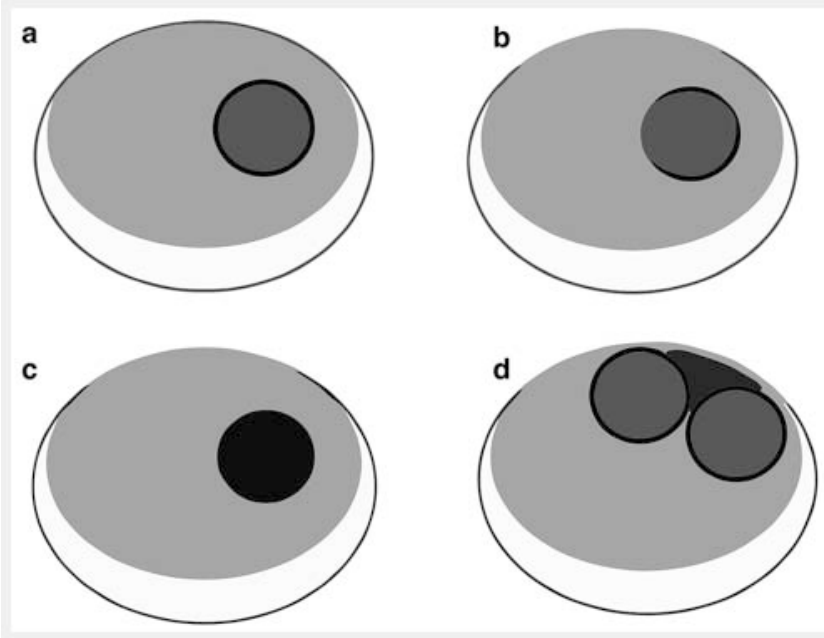

- Fig. 5 Schematic illustration of different types of atypical BPHnodules in comparison to the typical BPH-nodule.

\section{Interpretation of DWI}

Categories 2 and 3 of DWI were not clearly defined in version 2.0 and have occasionally resulted in difficulties regarding image interpretation. PI-RADS 2.1 revised both of these categories (see - Fig. 3). Table 1 provides a comparison of the definitions of DWI categories 2 and 3 between PI-RADS versions 2 and 2.1.

The term "marked" indicates a greater signal deviation than at any other focus in the same zone. In the case of multiple lesions, according to PI-RADS 2.1 (analogous to version 2.0) up to four lesions in categories 3-5 can be specified with the index lesion corresponding to the one with the highest PI-RADS score or, in the case of equivalent lesions, to the one with extraprostatic extension (if not applicable, the largest lesion).

\section{Interpretation of DCE}

While the definition of positive contrast enhancement in DCE remains unchanged, PI-RADS v2.1 states with respect to negative contrast enhancement that diffuse or multifocal enhancement is not a typical sign of cancer but rather is an expression of inflammatory changes. Focal enhancement of a lesion demonstrating typical features of BPH on $\mathrm{T} 2 \mathrm{~W}$ is still not considered a positive finding.

\section{Prostate volume measurement}

Every mpMRI finding in the prostate should include a volume measurement of the organ, in order to use this measurement to calculate the individual PSA density (e. g., cut-off $>0.15 \mathrm{ng} / \mathrm{ml} / \mathrm{cm}^{3}$ ) as a clinical biomarker of increasing importance for clinical decision processes [46, 47]. A new recommendation in PI-RADS 2.1 is to measure the largest anterior-posterior (ap) extension of the prostate on a mid-sagittal image (not axial as previously recommended). The goal here is to measure the ap extension independent of the orientation of the axial plane perpendicular to the long axis of the prostate (perpendicular to the max. extension of the prostate between the base and apex on the sagittal plane). Methods for the 

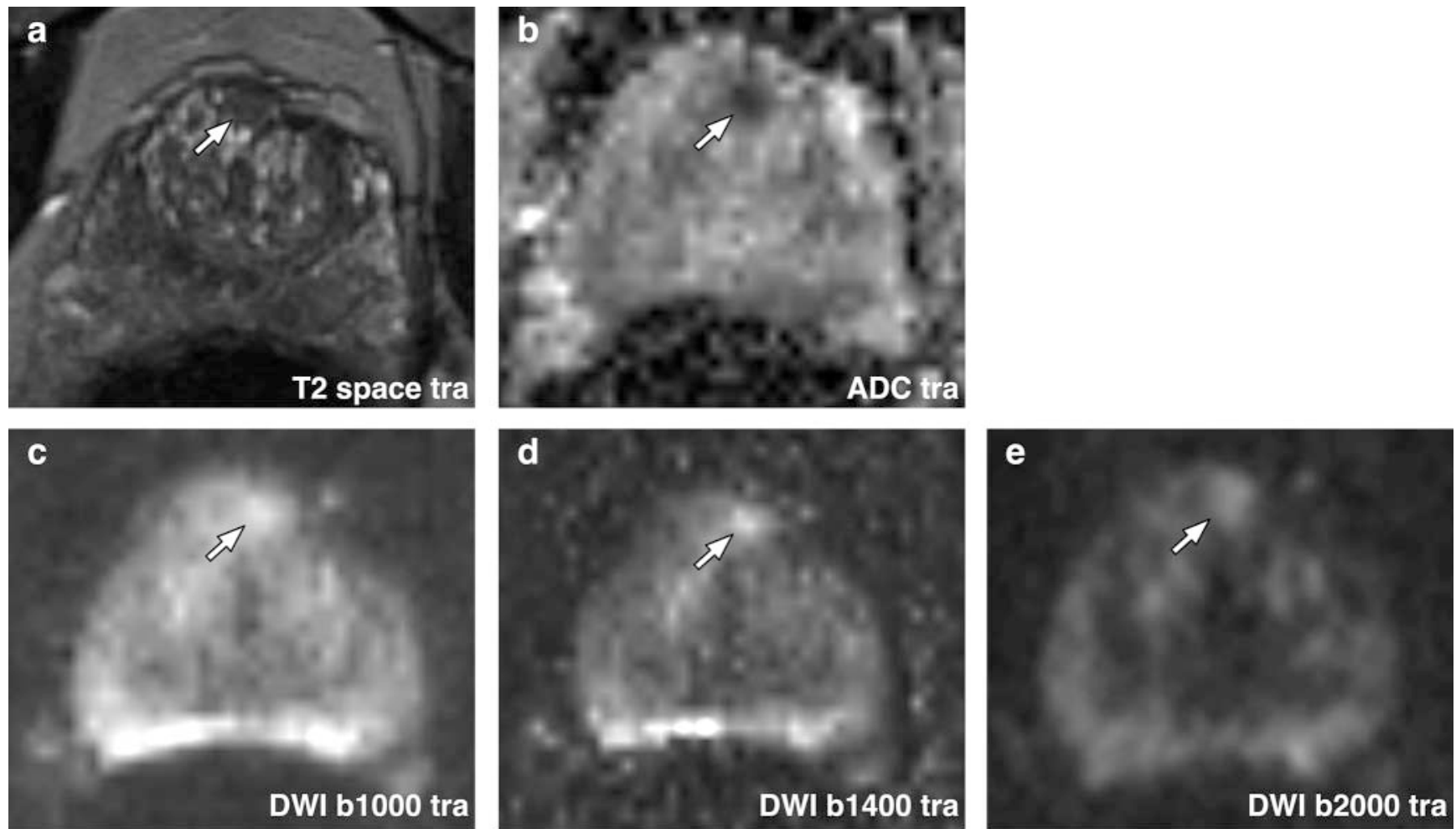

> Fig. 6 Upgrade from a PI-RADS-2 to a PI-RADS-3 lesion in a 62-year-old man with a PSA of $6.08 \mathrm{ng} / \mathrm{ml}$. Circumscribed low T2w signal a without a capsule at the anterior margin of the TZ paramedian left, corresponding to a PI-RADS 2 in the T2 image ("atypical node"). In addition to a significant signal drop in the ADC maps $\mathbf{b}$, a corresponding significant diffusion restriction is shown in the b1000 image $\mathbf{c}$, in the b1400 image $\mathbf{d}$ and in the b2000 image e, corresponding to a PI-RADS score 4 in the DWI. According to PI-RADS 2.1, this results in an overall PI-RADS score of 3.

- Table 1 Changes in definitions of PI-RADS categories 2 and 3 in diffusion-weighted imaging in PI-RADS v2.1 compared to PI-RADS v2.0.

\begin{tabular}{|l|l|l|}
\hline & PI-RADS 2.1 & PI-RADS 2.0 \\
\hline $\begin{array}{l}\text { DWI PI-RADS } \\
\text { category 2 }\end{array}$ & $\begin{array}{l}\text { linear/wedge-shaped hypoin- } \\
\text { tense on ADC and/or linear/ } \\
\text { wedge-shaped hyperintense } \\
\text { on high b-value images } \\
\text { (no focal lesions) }\end{array}$ & $\begin{array}{l}\text { in Fig. 7) } \\
\text { intense on ADC }\end{array}$ \\
\hline $\begin{array}{l}\text { DWI PI-RADS } \\
\text { category 3 }\end{array}$ & $\begin{array}{l}\text { focal (discrete and different } \\
\text { from the background) } \\
\text { hypointense on ADC and/or } \\
\text { focal hyperintense on high } \\
\text { b-value DWI; may be mark- } \\
\text { edly hypointense on ADC or } \\
\text { markedly hyperintense on } \\
\text { high b-value DWI, but not } \\
\text { both }\end{array}$ & $\begin{array}{l}\text { focal mildly/moder- } \\
\text { ately hypointense on } \\
\text { ADC and isointense/ } \\
\text { mildly hyperintense } \\
\text { on highalue }\end{array}$ \\
\hline
\end{tabular}

automatic segmentation of the prostate are also considered as an alternative [17].

\section{Prostate sector map}

The prostate sector map is intended to make it easier to allocate described and classified lesions as precisely as possible to an anatomical region of the prostate. 27 prostate sectors were defined for this purpose in the first PI-RADS version and 36 in the second version. PI-RADS 2.1 has introduced two additional sectors so that the sector map of the prostate includes 38 prostate sectors, two sectors for the seminal vesicles, and one sector for the membranous urethra for a total of 41 sectors. The posteromedial zones of the peripheral zone (PZpm) to the left and right of the base of the prostate are a new addition to PI-RADS 2.1.

\section{Summary}

PI-RADS version 2.1 addresses various small changes regarding examination technique and interpretation of findings and is an evolution of PI-RADS 2.0 with the basic concept still being zonerelated dominant sequences.

The greater importance placed on diffusion imaging in the transition zone with the goal of increasing the still comparably low sensitivity of mpMRI in this zone is particularly relevant. The newly introduced differentiation between "typical" and "atypical" $\mathrm{BPH}$ nodules based on $\mathrm{T} 2$ sequences in the transition zone places an even greater focus on this zone. The introduction of the mandatory ultra-high b-value in DWI with which many centers have already had good experiences is also a welcome addition. Given the increase in discussions regarding biparametric MRI, it is fitting for PI-RADS version 2.1 to address this option. However, multiparametric MRI remains the method of choice, particularly at facilities with a small number of cases. 

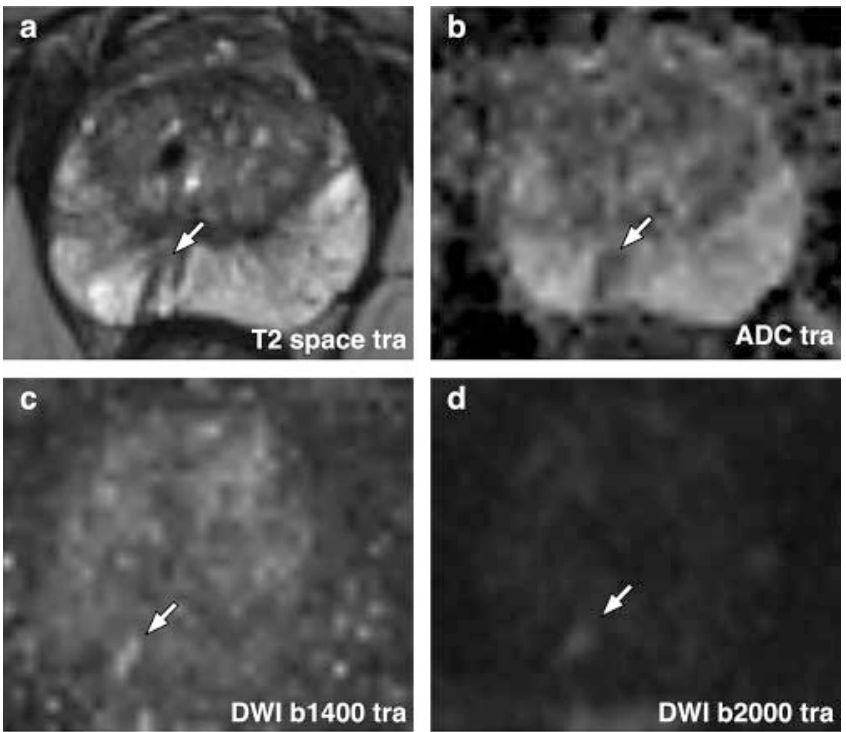

- Fig. 7 Typical PI-RADS-2 lesion in DWI in a 50-year-old biopsynaïve man with an increase in PSA from $3.76 \mathrm{ng} / \mathrm{ml}$ to $4.17 \mathrm{ng} / \mathrm{ml}$ within 5 months. According to PI-RADS 2.1, this is a linear, band/ wedge-shaped, non-focal signal reduction in the posteromedial right PZ (arrow) in T2w a with corresponding diffusion restriction in $\mathrm{ADC}$ maps $\mathbf{b}$ and corresponding signal increase in both the b1400 image $\mathbf{c}$ and the b2000 image $\mathbf{d}$.

Moreover, simplification of the scoring algorithm, detailed definition of specific signal changes, and further standardization of image acquisition are intended to help lower interreader variability and to further strengthen the value of mpMRI in the detection of clinically significant prostate cancer.

\section{Outlook}

PI-RADS is not a fixed concept but rather is subject to constant change. Future versions must address clinical and technical advances. Due to the good experiences regarding the detection and localization of prostate cancer, there has been increasing interest in the applicability of PI-RADS or other scoring systems for active surveillance and in the post-therapeutic setting. As a result of rapid advances, artificial intelligence and deep learning algorithms will probably support the interpretation of findings in the future [48]. It may be possible in the future with the help of new qualitative and quantitative factors still to be evaluated to link the PI-RADS system, like the BI-RADS method, to concrete instructions regarding biopsy to verify findings with the incorporation of additional clinical and primarily laboratory parameters (e. g. PCA-3, etc.) [49].

\section{Conflict of Interest}

The authors declare that they have no conflict of interest.
References

[1] Bray F, Ferlay J, Soerjomataram I et al. Global cancer statistics 2018: GLOBOCAN estimates of incidence and mortality worldwide for 36 cancers in 185 countries. CA Cancer J Clin 2018; 68: 394-424. doi:10.3322/caac. 21492

[2] Lam TBL, MacLennan S, Willemse PM et al. EAU-EANM-ESTRO-ESURSIOG Prostate Cancer Guideline Panel Consensus Statements for Deferred Treatment with Curative Intent for Localised Prostate Cancer from an International Collaborative Study (DETECTIVE Study). Eur Urol 2019; 76: 790-813. doi:10.1016/j.eururo.2019.09.020

[3] Kim R, Kim CK, Park JJ et al. Prognostic Significance for Long-Term Outcomes Following Radical Prostatectomy in Men with Prostate Cancer: Evaluation with Prostate Imaging Reporting and Data System Version 2. Korean J Radiol 2019; 20: 256-264. doi:10.3348/kjr.2018.0613

[4] Schlemmer HP. Multiparametrische MR-Bildgebung beim Prostatakarzinom. Radiologie up2date 2017; 17: 43-60. doi:10.1055/s-0042-122604

[5] Barentsz JO, Richenberg J, Clements R et al. ESUR prostate MR guidelines 2012. Eur Radiol 2012; 22: 746-757. doi:10.1007/s00330-011-2377-y

[6] Weinreb JC, Barentsz JO, Choyke PL et al. PI-RADS Prostate Imaging Reporting and Data System: 2015, Version 2. Eur Urol 2016; 69: 16-40. doi:10.1016/j.eururo.2015.08.052

[7] Rosenkrantz AB, Ginocchio LA, Cornfeld D et al. Interobserver Reproducibility of the PI-RADS Version 2 Lexicon: A Multicenter Study of Six Experienced Prostate Radiologists. Radiology 2016; 280: 793-804. doi:10.1148/radiol.2016152542

[8] Borofsky S, George AK, Gaur S et al. What Are We Missing? False-Negative Cancers at Multiparametric MR Imaging of the Prostate. Radiology 2018; 286: 186-195. doi:10.1148/radiol.2017152877

[9] Kido A, Tamada T, Kanomata $\mathrm{N}$ et al. Multidimensional analysis of clinicopathological characteristics of false-negative clinically significant prostate cancers on multiparametric MRI of the prostate in Japanese men. Jpn J Radiol 2019; 37: 154-164. doi:10.1007/s11604-018-0801-9

[10] Muller BG, Shih JH, Sankineni S et al. Prostate Cancer: Interobserver Agreement and Accuracy with the Revised Prostate Imaging Reporting and Data System at Multiparametric MR Imaging. Radiology 2015; 277 : 741-750. doi:10.1148/radiol.2015142818

[11] Rosenkrantz AB, Oto A, Turkbey B et al. Prostate Imaging Reporting and Data System (PI-RADS), Version 2: A Critical Look. Am J Roentgenol 2016; 206: 1179-1183. doi:10.2214/AJR.15.15765

[12] Turkbey B, Rosenkrantz AB, Haider MA et al. Prostate Imaging Reporting and Data System Version 2.1: 2019 Update of Prostate Imaging Reporting and Data System Version 2. Eur Urol 2019; 76: 340-351. doi:10.1016/j.eururo.2019.02.033

[13] Barrett T, Rajesh A, Rosenkrantz AB et al. PI-RADS version 2.1: one small step for prostate MRI. Clin Radiol 2019; 74: 841-852. doi:10.1016/ j.crad.2019.05.019

[14] Le Bihan D, Breton E, Lallemand D et al. Separation of diffusion and perfusion in intravoxel incoherent motion MR imaging. Radiology 1988; 168: 497-505. doi:10.1148/radiology.168.2.3393671

[15] Barrett T, Priest AN, Lawrence EM et al. Ratio of Tumor to Normal Prostate Tissue Apparent Diffusion Coefficient as a Method for Quantifying DWI of the Prostate. Am J Roentgenol 2015; 205: W585-W593. doi:10.2214/AJR.15.14338

[16] Jensen JH, Helpern JA, Ramani A et al. Diffusional kurtosis imaging: the quantification of non-gaussian water diffusion by means of magnetic resonance imaging. Magn Reson Med 2005; 53: 1432-1440. doi: $10.1002 / \mathrm{mrm} .20508$

[17] Rosenkrantz AB, Sigmund EE, Johnson G et al. Prostate cancer: feasibility and preliminary experience of a diffusional kurtosis model for detection and assessment of aggressiveness of peripheral zone cancer. Radiology 2012; 264: 126-135. doi:10.1148/radiol.12112290 
[18] DeLano MC, Cooper TG, Siebert JE et al. High-b-value diffusion-weighted MR imaging of adult brain: image contrast and apparent diffusion coefficient map features. AJNR Am J Neuroradiol 2000; 21: 1830-1836

[19] Kim CK, Park BK, Kim B. High-b-value diffusion-weighted imaging at $3 \mathrm{~T}$ to detect prostate cancer: comparisons between $b$ values of 1000 and 2000 s/mm². Am J Roentgenol 2010; 194: W33-W37. doi:10.2214/ AJR.09.3004

[20] Agarwal HK, Mertan FV, Sankineni S et al. Optimal high b-value for diffusion weighted MRI in diagnosing high risk prostate cancers in the peripheral zone. J Magn Reson Imaging 2017; 45: 125-131. doi:10.1002/jmri.25353

[21] Syer T], Godley KC, Cameron D et al. The diagnostic accuracy of high b-value diffusion- and T2-weighted imaging for the detection of prostate cancer: a meta-analysis. Abdom Radiol (NY) 2018; 43: 17871797. doi:10.1007/s00261-017-1400-4

[22] Metens T, Miranda D, Absil J et al. What is the optimal b value in diffusion-weighted MR imaging to depict prostate cancer at 3T. Eur Radiol 2012; 22: 703-709. doi:10.1007/s00330-011-2298-9

[23] Ohgiya Y, Suyama J, Seino $N$ et al. Diagnostic accuracy of ultra-high-b-value 3.0-T diffusion-weighted MR imaging for detection of prostate cancer. Clin Imaging 2012; 36: 526-531. doi:10.1016/j.clinimag.2011.11.016

[24] Jambor I. Optimization of prostate MRI acquisition and post-processing protocol: a pictorial review with access to acquisition protocols. Acta Radiol Open 2017; 6: 2058460117745574 doi:10.1177| 2058460117745574

[25] Panebianco V, Giganti F, Kitzing YX et al. An update of pitfalls in prostate mpMRI: a practical approach through the lens of PI-RADS v. 2 guidelines. Insights Imaging 2018; 9: 87-101. doi:10.1007/s13244-017-0578-x

[26] Ream JM, Doshi AM, Dunst D et al. Dynamic contrast-enhanced MRI of the prostate: An intraindividual assessment of the effect of temporal resolution on qualitative detection and quantitative analysis of histopathologically proven prostate cancer. J Magn Reson Imaging 2017; 45: 1464-1475. doi:10.1002/jmri.25451

[27] Boesen L, Nørgaard N, Løgager V et al. Assessment of the Diagnostic Accuracy of Biparametric Magnetic Resonance Imaging for Prostate Cancer in Biopsy-Naive Men: The Biparametric MRI for Detection of Prostate Cancer (BIDOC) Study. JAMA Netw Open 2018; 1: e180219 doi:10.1001/jamanetworkopen.2018.0219

[28] Jambor I, Boström PJ, Taimen P et al. Novel biparametric MRI and targeted biopsy improves risk stratification in men with a clinical suspicion of prostate cancer (IMPROD Trial). J Magn Reson Imaging 2017; 46: 10891095. doi:10.1002/jmri.25641

[29] Gupta RT, Mehta KA, Turkbey B et al. PI-RADS: Past, present, and future. J Magn Reson Imaging 2019. doi:10.1002/jmri.26896

[30] Greer MD, Shih JH, Lay N et al. Validation of the Dominant Sequence Paradigm and Role of Dynamic Contrast-enhanced Imaging in PI-RADS Version 2. Radiology 2017; 285: 859-869. doi:10.1148/radiol.2017161316

[31] Krishna S, McInnes M, Lim C et al. Comparison of Prostate Imaging Reporting and Data System versions 1 and 2 for the Detection of Peripheral Zone Gleason Score 3 + 4 = 7 Cancers. Am J Roentgenol 2017; 209: W365-W373. doi:10.2214/AJR.17.17964

[32] Rosenkrantz AB, Babb JS, Taneja SS et al. Proposed Adjustments to PI-RADS Version 2 Decision Rules: Impact on Prostate Cancer Detection. Radiology 2017; 283: 119-129. doi:10.1148/radiol.2016161124

[33] Caglic I, Barrett T. Optimising prostate mpMRI: prepare for success. Clin Radiol 2019; 74: 831-840. doi:10.1016/j.crad.2018.12.003
[34] Iwazawa J, Mitani T, Sassa S et al. Prostate cancer detection with MRI: is dynamic contrast-enhanced imaging necessary in addition to diffusionweighted imaging. Diagn Interv Radiol 2011; 17: 243-248. doi:10.4261/ 1305-3825.DIR.3605-10.1

[35] Padhani AR, Weinreb J, Rosenkrantz AB et al. Prostate Imaging-Reporting and Data System Steering Committee: PI-RADS v2 Status Update and Future Directions. Eur Urol 2019; 75: 385-396. doi:10.1016/ j.eururo.2018.05.035

[36] Scialpi M, Martorana E, Scialpi P et al. Round table: arguments in supporting abbreviated or biparametric MRI of the prostate protocol. Abdom Radiol (NY) 2020. doi:10.1007/s00261-020-02510-w

[37] Scialpi M, Aisa MC, D‘Andrea A et al. Simplified Prostate Imaging Reporting and Data System for Biparametric Prostate MRI: A Proposal. Am J Roentgenol 2018; 211: 379-382. doi:10.2214/AJR.17.19014

[38] Vargas HA, Akin O, Franiel T et al. Normal central zone of the prostate and central zone involvement by prostate cancer: clinical and MR imaging implications. Radiology 2012; 262: 894-902. doi:10.1148/ radiol. 11110663

[39] Hansford BG, Karademir I, Peng Y et al. Dynamic contrast-enhanced MR imaging features of the normal central zone of the prostate. Acad Radiol 2014; 21: 569-577. doi:10.1016/j.acra.2014.01.013

[40] Cohen RJ, Shannon BA, Phillips M et al. Central zone carcinoma of the prostate gland: a distinct tumor type with poor prognostic features. J Urol 2008; 179: 1762-1767; discussion 1767. doi:10.1016/j.juro. 2008.01.017

[41] Ward E, Baad M, Peng Y et al. Multi-parametric MR imaging of the anterior fibromuscular stroma and its differentiation from prostate cancer. Abdom Radiol (NY) 2017; 42: 926-934. doi:10.1007/s00261-016-0951-0

[42] Bouyé S, Potiron E, Puech P et al. Transition zone and anterior stromal prostate cancers: zone of origin and intraprostatic patterns of spread at histopathology. Prostate 2009; 69: 105-113. doi:10.1002/pros.20859

[43] McNeal JE, Redwine EA, Freiha FS et al. Zonal distribution of prostatic adenocarcinoma. Correlation with histologic pattern and direction of spread. Am J Surg Pathol 1988; 12: 897-906. doi:10.1097/00000478198812000-00001

[44] Kayhan A, Fan X, Oommen J et al. Multi-parametric MR imaging of transition zone prostate cancer: Imaging features, detection and staging. World J Radiol 2010; 2: 180-187. doi:10.4329/wjr.v2.i5.180

[45] Tamada T, Kido A, Takeuchi M et al. Comparison of PI-RADS version 2 and PI-RADS version 2.1 for the detection of transition zone prostate cancer. Eur J Radiol 2019; 121: 108704 doi:10.1016/j.ejrad.2019.108704

[46] Søndergaard G, Vetner M, Christensen PO. Periferal cystic hyperplasia of the prostate gland. Acta Pathol Microbiol Immunol Scand A 1987; 95: 137-139. doi:10.1111/j.1699-0463.1987.tb00020_95a.x

[47] Hansen NL, Barrett T, Koo B et al. The influence of prostate-specific antigen density on positive and negative predictive values of multiparametric magnetic resonance imaging to detect Gleason score 7-10 prostate cancer in a repeat biopsy setting. BJU Int 2017; 119: 724-730. doi:10.1111/bju.13619

[48] Bonekamp D, Kohl S, Wiesenfarth M et al. Radiomic Machine Learning for Characterization of Prostate Lesions with MRI: Comparison to ADC Values. Radiology 2018; 289: 128-137. doi:10.1148/radiol.2018173064

[49] Kaufmann S, Bedke J, Gatidis S et al. Prostate cancer gene 3 (PCA3) is of additional predictive value in patients with PI-RADS grade III (intermediate) lesions in the MR-guided re-biopsy setting for prostate cancer. World J Urol 2016; 34: 509-515. doi:10.1007/s00345-015-1655-8 